\title{
Annexin A9 promotes invasion and metastasis of colorectal cancer and predicts poor prognosis
}

\author{
SUYANG YU $^{1}$, HONGLEI BIAN ${ }^{1}$, XIN GAO $^{2}$ and LIN GUI ${ }^{1}$ \\ ${ }^{1}$ First Department of Colorectal Surgery, The Third Hospital of Hebei Medical University; \\ ${ }^{2} \mathrm{ICU}$ of Department of Anesthesiology, The Second Hospital of Hebei Medical University, \\ Shijiazhuang, Hebei 050000, P.R. China
}

Received June 20, 2017; Accepted January 8, 2018

DOI: $10.3892 / \mathrm{ijmm} .2018 .3432$

\begin{abstract}
Annexin A9 (ANXA9), a member of annexin family, has been reported be associated with colorectal cancer (CRC) carcinogenesis. However, the clinical significance of ANXA9 in CRC, particularly its correlation to invasion and metastasis remains ambiguous. The aim of the present study was to investigate the significance of ANXA9 in CRC and understand the molecular mechanism of ANXA9 in CRC invasion and metastasis. Expression levels of the ANXA9 protein in CRC tissues were detected using immunohistochemistry (IHC), and the clinical and prognostic value of ANXA9 was investigated. ANXA9-siRNA was utilized to investigate the effect and molecular mechanism of ANXA9 in HCT116 cells. The IHC result demonstrated that the positivity rate of the ANXA9 protein in CRC tissue was significantly higher than that in adjacent mucosa $(\mathrm{P}<0.05)$, which was consistent with the western blot results. ANXA9 protein expression levels are associated with invasion depth and lymphatic metastasis. Furthermore, patients with ANXA9-positive expression demonstrated a poor prognosis and ANXA9 was an independent risk factor for survival $(\mathrm{P}<0.05)$. After inhibiting ANXA9 in HCT116 cells, the activity and metastatic and invasion capacity of cells decreased significantly, and expression levels of ADAM metallopeptidase domain 17 and matrix metallopeptidase 9 were significantly downregulated, while the expression levels of tissue inhibitors of metalloproteinases-1 and E-cadherin were upregulated $(\mathrm{P}<0.05)$. Thus, positive ANXA9 expression may present as a novel marker for predicting poor prognosis in CRC patients, and ANXA9 may promote the invasion and metastasis of CRC by regulating invasion and metastasis-associated genes.
\end{abstract}

Correspondence to: Professor Honglei Bian, First Department of Colorectal Surgery, The Third Hospital of Hebei Medical University, 139 Ziqiang Road, Shijiazhuang, Hebei 050000, P.R. China E-mail: honglei_bian3815@163.com

Key words: Annexin A9, colorectal cancer, invasion, metastasis, prognosis, gene interference

\section{Introduction}

Colorectal cancer (CRC), the third most common malignancy worldwide (1), has been treated using combination therapy, including surgery, radiotherapy, chemotherapy and targeted drugs. However, a high proportion of CRC patients were diagnosed in the advanced stage (2), which resulted in poor prognosis and recurrence or metastasis even after treatment. Multiple genes are responsible for this process (3-8); however, the molecular mechanism remains unclear. Therefore, identifying novel genes is considered to be vital for analyzing pathophysiological variations, evaluating medical conditions and defining novel targets in CRC.

Annexins are a group of $\mathrm{Ca}^{2+}$-dependent phospholipid-binding proteins, of which the family members include A, B, C, D and E subgroups. In particular, numerous members of the Annexin A (ANXA) subgroup are closely associated with cancer development (9-11). ANXA9 is a family member of ANXA. A Japanese study demonstrated that expression of ANXA9 mRNA in CRC was associated with a poor prognosis (12), indicating that ANXA9 may be associated with CRC development. However, there are few studies regarding the molecular mechanism of ANXA9 in CRC. The aim of the present study was to investigate the value of ANXA9 protein detection in CRC evaluation and understand the mechanism of ANXA9 in CRC cells. Therefore, in the present study, expression levels of the ANXA9 protein were detected in clinical samples obtained from patients with CRC and the correlation between the ANXA9 protein and clinicopathologic features was analyzed. In addition, the prognosis of the CRC patients was recorded. Furthermore, the variations in CRC cell activity, invasion and migration were investigated under RNA interference by inhibiting ANXA9 expression in HCT116 cells and the alteration of associated genes [ADAM metallopeptidase domain 17 (ADAM17), matrix metallopeptidase 9 (MMP-9), tissue inhibitors of metalloproteinases-1 (TIMP-1), E-cadherin and $\mathrm{N}$-cadherin] was also detected.

\section{Materials and methods}

Ethical approval. The study protocol was approved by the Medical Ethics Committee of the Third Hospital of Hebei Medical University (Shijiazhuang, China). All methods used in 
the present study were performed according to the International Ethical Guidelines for Biomedical Research Involving Human Subjects, and informed consent was obtained from all participants prior to the study.

Participant enrollment. A total of 105 CRC patients whose cancer was removed at The Third Hospital of Hebei Medical University were recruited between January and December 2010. The mean age was 56.36 \pm 9.19 year (range, 38-78 years) with 30 females and 75 males. All participants were first diagnosed with CRC without any other malignancies and the diagnosis was conformed by pathological examination. No participants had received radio- or chemotherapy or targeted therapy prior to surgery. Complete clinicopathological data and follow-ups were recorded. TNM Classification of Malignant Tumors was performed according to Union for International Cancer Control/American Joint Committee on Cancer gastric cancer staging system (13). The follow-ups ended in December 2015. Paraffin-embedded samples of tumor tissues and adjacent mucosa ( $\geq 2 \mathrm{~cm}$ from the edge of the caner with no cancer cells verified) were obtained for the detection of ANXA9 protein expression. Additional fresh samples of tumor tissues and adjacent mucosa were collected from 20 participants who had undergone surgery at The Third Hospital of Hebei Medical University (Shijiazhuang, China) between March and October 2016. These samples were maintained at $-80^{\circ} \mathrm{C}$ and the expression level of ANXA9 proteins were detected by western blotting.

Cell lines and reagents. Human CRC cell lines, Caco-2, HCT116, SW620, SW480 and $\mathrm{L}_{\mathrm{O}} \mathrm{V}_{\mathrm{O}}$ were purchased from the Cell Resource Center of Life Sciences (Shanghai, China). Cells from the HT-29 cell line were preserved and incubated at the Scientific Research Center of The Third Hospital of Hebei Medical University (Shijiazhuang, China). Lipofectamine $^{\mathrm{TM}} 2000$ Reagent (Invitrogen; Thermo Fisher Scientific, Inc., USA), Gibco Dulbecco's modified Eagle's medium (DMEM) and Gibco fetal bovine serum (FBS; Thermo Fisher Scientific, Inc.) were applied. In addition, Thiazolyl Blue Tetrazolium Bromide (MTT), TRIzol (Invitrogen; Thermo Fisher Scientific, Inc.), and the quantitative polymerase chain reaction (qPCR) and protein extraction kits (cat.no. PROTTOT) were obtained from Sigma-Aldrich (Merck KGaA, Darmstadt, Germany). The PCR primers and ANXA9-siRNA were designed and synthesized by Sangon Biotech, Co., Ltd. (Shanghai, China). ANXA9 (cat. no. sc-373934), ADAM17 (cat. no. sc-390859), MMP-9 (cat. no. sc-12759), TIMP-1 (cat. no. sc-365905), E-cadherin (cat. no. sc-71008), N-cadherin (cat. no. sc-59987) and $\beta$-actin (cat. no. sc-8432) antibodies were obtained from Santa Cruz Biotechnology, Inc. (Dallas, TX, USA).

IHC staining assay. Paraffin specimens were deparaffinized and rehydrated, and immunohistochemical staining of surfactant proteins (S-P) (cat. no. sc-80621) was performed according to the manufacturer's instruction (Santa Cruz Biotechnology, Inc.) Five random visual fields (magnification, $\mathrm{x} 400 ; 100$ cells per field) for each section were evaluated by pathologists. Expression levels of the ANXA9 protein were determined as positive if yellow or brown plasmids were observed in the cytoplasm or on the cell membrane. Expression positivity was scored as follows: i) Darkness of staining (transparent, 0; light yellow, 1; brownish-yellow, 2 ; and brown, 3 ; ii) ratio of positive to negative cells (positivity of $0 \%, 0 ; \leq 10 \%, 1 ; 11-50 \%, 2 ; 51-75 \%, 3$; and $>75 \%$ ). The two scores were added and $\leq 2$ was considered to be negative (-) and $>2$ was considered to be positive $(+)$.

Western blot assay. Tissue and cell lysates were prepared with lysis buffer [1\% Triton X-100, $150 \mathrm{mM} \mathrm{NaCl,} 10 \mathrm{mM}$ Tris- $\mathrm{HCl}$ (pH 7.4), 1 mM ethylene diamine tetraacetic acid (EDTA), $1 \mathrm{mM}$ ethylene glycol-bis( $\beta$-aminoethylether)tetraacetic acid ( $\mathrm{pH} 8.0$ ), $0.2 \mathrm{mM} \mathrm{Na}_{3} \mathrm{VO}_{4}, 0.2 \mathrm{mM}$ phenylmethylsulfonyl fluoride, and $0.5 \% \mathrm{NP}-40]$. The samples were rinsed in ice-cold lysis buffer for $20 \mathrm{~min}$ followed by centrifugation for $10 \mathrm{~min}$ at $7,104 \mathrm{x} \mathrm{g}$ at $4^{\circ} \mathrm{C}$. The supernatant was collected and the bicinchoninic acid assay was performed for quantitation of protein. Equal quantities of protein $(60 \mu \mathrm{g})$ from each sample were separated in $10 \%$ dodecyl sulfate, sodium salt-polyacrylamide gel electrophoresis gels and electrotransferred to polyvinylidene difluoride membranes (100 V, $2 \mathrm{~h}$ ). The membranes were blocked in 5\% non-fat milk for $2 \mathrm{~h}$ at room temperature, followed by incubation in diluted antibodies at $4{ }^{\circ} \mathrm{C}$ overnight. The following antibodies were used: Mouse anti-ANXA9 (1:200, cat. no. sc-373934), mouse anti-ADAM17 (1:200, cat. no. sc-390859), mouse anti-MMP-9 (1:400, cat. no. sc-12759), mouse anti-TIMP-1 (1:200, cat. no. sc-365905), mouse anti-E-cadherin (1:200, cat. no. sc-71008), mouse anti-N-cadherin (1:800, cat. no. sc-59987), mouse anti- $\beta$-actin (1:200, cat. no. sc-8432) all Santa Cruz Biotechnology, Inc. Following three rinses with Tris- $\mathrm{Hcl}, \mathrm{NaCl}$ and Tween-20 (TBST), blots were incubated with peroxidase-conjugated donkey anti-mouse antibody (1:2,000; cat. no. AB10085; Jackson ImmunoResearch Laboratories, Inc., West Grove, PA, USA) for $2 \mathrm{~h}$ at room temperature. After three rinses with TBST and one with TBS, the optical density (OD) of the bands was detected using an enhanced chemiluminescence detection system. The concentration of proteins in the samples was then determined by comparing the OD of the samples to the standard curve.

Cell culture. All cell lines were cultured in DMEM supplemented with $10 \% \mathrm{FBS}, 100 \mathrm{U} / \mathrm{ml}$ penicillin and $0.1 \mathrm{mg} / \mathrm{ml}$ streptomycin. Cells were maintained at $37^{\circ} \mathrm{C}$ in an incubator saturated with $5 \% \mathrm{CO}_{2}$. Cells were dissociated with $0.25 \%$ trypsin containing $0.02 \%$ EDTA and were passaged. Cells in the exponential growth phase were used for the experiments.

ANXA9-siRNA transfection. Design and synthesis of the sequence of siRNA targeting ANXA9 was performed as below: siRNA, 5'-GCAGUCUACAAACACAAUUtt-3' and non-specific control siRNA (NS-siRNA), 5'-UUCUCC GAACGUGUCACGUtt-3'. HCT116 cells were transplanted into 6-well plates $24 \mathrm{~h}$ prior to transfection (density of $1 \times 10^{6}$ cells $/ \mathrm{ml}$ ). Plasmid transfection was performed using Lipofectamine $^{\mathrm{TM}} 2000$ according to the manufacturer's instructions after samples were washed with serum- and antibody-free DMEM. Efficiency of transfection and ANXA9 suppression was detected $24 \mathrm{~h}$ after transfection. Experimental samples were divided into three groups according to the 
Table I. Primer sequences for quantitative polymerase chain reaction.

\begin{tabular}{lll}
\hline Gene & \multicolumn{1}{c}{ Forward primer (5' to 3') } & \multicolumn{1}{c}{ Reverse primer (5' to 3') } \\
\hline Annexin A9 & TGAGCCCAATTACCAAGTCC & GTTCAGCCAAACACGGAAAT \\
ADAM metallopeptidase domain 17 & ATCAAACCCTTCCTGCG & CAAACCCATCCTCGTCCA \\
Matrix metallopeptidase 9 & AGAACCAATCTCACCGACAGG & CGACTCTCCACGCATCTCT \\
Tissue inhibitors of metalloproteinases-1 & ACTTCCACAGGTCCCACAAC & GCATTCCTCACAGCCAACAG \\
GAPDH & GACCCCTTCATTGACCTCAAC & CGCTCCTGGAAGATGGTGAT \\
\hline
\end{tabular}

transfection status, which were the Lipofectamine ${ }^{\mathrm{TM}} 2000$ transfected group (blank group), NS-siRNA transfected group (NS-siRNA group) and the ANXA9-siRNA transfected group (ANXA9-siRNA group).

RNA extraction and $q P C R$. Total cellular RNA in the tissue specimens and cells of different groups was extracted using TRIzol (Invitrogen; Thermo Fisher Scientific, Inc.) according to the manufacturer's protocol. qPCR was performed in a total volume of $20 \mu \mathrm{l}$ containing $1 \mu \mathrm{l}$ reverse transcription product as a template for PCR, 2X UltraSYBR mixture (10 $\mu \mathrm{l}$; Applied Biosystems; Thermo Fisher Scientific, Inc.), $10 \mu \mathrm{mol} / 1$ per $1 \mu \mathrm{l}$ primer, $8 \mu \mathrm{l}$ DNase/RNase-Free water. The primer sequences used in PCR are presented in Table I. PCR was performed over 35 cycles as follows: Initial denaturation at $95^{\circ} \mathrm{C}$ for $5 \mathrm{~min}$, denaturation at $95^{\circ} \mathrm{C}$ for $30 \mathrm{sec}$, annealing at $60^{\circ} \mathrm{C}$ for $30 \mathrm{sec}$ and elongation at $72^{\circ} \mathrm{C}$ for $30 \mathrm{sec}$. Fluorescence was detected at the end of each cycle. The specificity of the products was confirmed by melting curve analysis. GAPDH served as an endogenous reference to standardize relative expression levels for data analysis to calculate the expression levels.

MTT assay. Cells were incubated in 96-well plates at a density of $1 \times 10^{5}$ cells $/ \mathrm{ml}$. When the cell density reached $70-80 \%$ confluence, ANXA9-siRNA or NS-siRNA was transfected. The cells were plated in 6 replicate wells per cell density. Following incubation at $37^{\circ} \mathrm{C}$ for $20 \mathrm{~h}, 20 \mu \mathrm{l}(5 \mathrm{mg} / \mathrm{ml}) \mathrm{MTT}$ was added for another 4-h incubation and discarded, followed by the addition of $150 \mu \mathrm{l}$ DMSO in each well and gentle shaking at room temperature for $15 \mathrm{~min}$. The OD value was measured at a wavelength of $490 \mathrm{~nm}$ using a microplate spectrophotometer. Each treatment was performed in triplicate.

Wound healing assay. HCT116 cells were formed into monolayer suspension (density, $1 \times 10^{6}$ cell $/ \mathrm{ml}$ ) and seeded in each well of 6-well culture plates. The cells were transfected with ANXA9-siRNA or NS-siRNA at $60-70 \%$ confluence and cultured until $100 \%$. The culture medium was removed and the cells were rinsed with phosphate-buffered saline (PBS). This monolayer was then scored using a sterile pipette tip to form scratches and was rinsed with PBS again to remove any dislodged cells. Wound closure was visualized using a microscope. The procedures were performed in triplicate.

Transwell assay. Each well in the upper chamber was coated with $100 \mu$ l Matrigel (BD Biosciences, Franklin Lakes, NJ, USA) under ultraviolet light. HCT116 cells were suspended and plated at $1 \times 10^{6}$ cells $/ \mathrm{ml}$ in 6 -well plates. These cells were cultured until 60-70\% confluence for transfection. After a $24-\mathrm{h}$ incubation at $37^{\circ} \mathrm{C}, 200 \mu \mathrm{l}$ cells were extracted from each group and plated in the upper chamber of a Transwell, while DMEM was added to the lower chamber. After removing any excess Matrigel and non-invading cells from the upper chamber, the Transwell membranes were fixed in methanol for $10 \mathrm{~min}$ and stained with crystal violet for $30 \mathrm{~min}$ at $37^{\circ} \mathrm{C}$. Cells on the underside of the membranes that had invaded the Matrigel were counted under an inverted microscope (Carl Zeiss AG, Oberkochen, Germany). The treatment was repeated three times.

Statistical analysis. All of the data was analyzed by SPSS 26.0 statistical software (IBM Corp., Armonk, NY, USA). Quantitative data was represented as the mean \pm standard deviation, and the deviation between groups was analyzed using one way analysis and Dunnett t-test and variation analysis. Categorical data were expressed as percentages and analyzed using the $\chi^{2}$ test. Kaplan-Meier analysis and COX's proportional hazard regression model were utilized to investigate the prognostic factors of the ANXA9 protein. $\mathrm{P}<0.05$ was considered to indicate a statistically significant difference.

\section{Results}

Expression levels of ANXA9 in CRC tissues and adjacent mucosa. The IHC result demonstrated that the positive rate of ANXA9 protein expression in CRC tissue samples was higher than that in the adjacent mucosa with $76.19 \%(80 / 105)$, and $16.19 \%(17 / 105)$, respectively $\left(\chi^{2}=76.041 ; \mathrm{P}<0.001\right)$, as illustrated in Fig. 1A. Similarly, the result of western blot analysis demonstrated that the ANXA9 expression level was higher in CRC tissues compared with the adjacent mucosa $(\mathrm{P}<0.001)$ (Fig. 1B).

Association between expression levels of ANXA9 in CRC tissue and clinicopathological characteristics with CRC patients. The result demonstrated that a higher ANXA9 positive rate presented deeper-infiltration and lymphatic metastasis in the CRC tissue samples $(\mathrm{P}<0.05)$, and no significant correlation was identified between ANXA9 and other clinicopathological parameters $(\mathrm{P}>0.05)$. The results were illustrated in Table II.

Prognostic value of ANXA9 detection for CRC patients. The association between ANXA9 expression levels and 




a CRC tissues $(\times 100)$

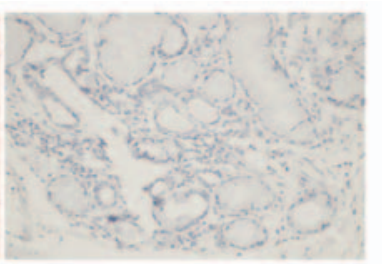

b Adjacent mucosa tissues $(\times 100)$

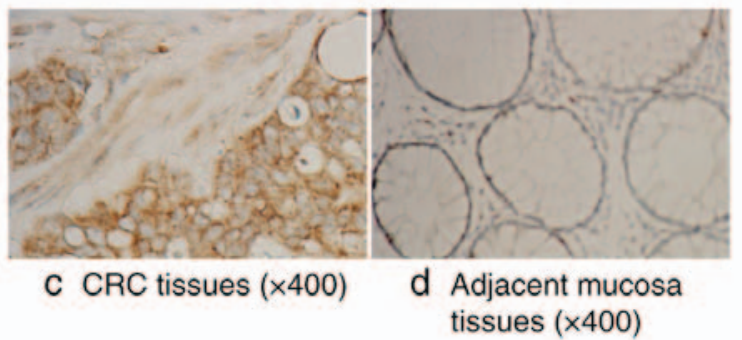

B


Figure 1. Expression level of ANXA9 protein in CRC and adjacent mucosa tissue samples. (A) Paraffin embedded tissues from 105 CRC patients were collected to detect ANXA9 protein expression by immunohistochemistry (magnification, $\mathrm{x} 400$ ). Positive staining of ANXA9 protein was located in the cell membrane and/or cytoplasm. (B) Fresh tissue samples of CRC and adjacent mucosa from another 20 patients were subjected to western blot assay for detection of ANXA9 protein expression levels. ${ }^{*} \mathrm{P}<0.05$ vs. CRC tissues. CRC, colorectal cancer; ANXA9, Annexin A9.

prognosis was analyzed and presented using a Kaplan-Meier

survival curve (Fig. 2). The data demonstrates that the overall survival rate was lower in the patients with positive ANXA9 expression compared with those with negative ANXA9 expression $(\mathrm{P}=0.005)$. According to Cox's proportional hazards regression model presented in Table III, the present study illustrated that ANXA9 expression level was an independent risk factor in CRC prognosis $(\mathrm{P}=0.022)$, and other independent risk factors, including lymphatic metastasis, differentiation and distant metastasis $(\mathrm{P}=0.017,0.021$ and 0.026 , respectively).

ANXA9 expression levels in CRC cell lines. As a result of western blotting, different levels of ANXA9 protein were detected in six CRC cell lines, among which the highest expression level of ANXA9 protein was demonstrated in HCT116 cells, and thus was selected for subsequent experiments (Fig. 3).
Table II. Association between ANXA9 protein and clinicopathological parameters in CRC patients $(\mathrm{n}=105)$.

\begin{tabular}{|c|c|c|}
\hline $\begin{array}{l}\text { Clinicopathological } \\
\text { parameter }\end{array}$ & $\begin{array}{c}\text { Positive } \\
(\mathrm{n}=80)\end{array}$ & $\begin{array}{l}\text { Negative } \\
(\mathrm{n}=25)\end{array}$ \\
\hline
\end{tabular}

Sex

$\begin{array}{lrrrr}\text { Male } & 59 & 16 & 0.887 & 0.346 \\ \text { Female } & 21 & 9 & & \end{array}$

Age (years)

$\begin{array}{lrrrr}\geq 60 & 28 & 6 & 1.053 & 0.305 \\ <60 & 52 & 19 & & \end{array}$

Tumor differentiation

$\begin{array}{lllll}\text { Well differentiated } & 53 & 19 & 0.840 & 0.359\end{array}$

Poorly differentiated $\quad 27 \quad 6$

Depth of invasion

$\begin{array}{lllll}\text { With serosal infiltration } & 56 & 11 & 5.576 & 0.018\end{array}$

Without serosal $\quad 24 \quad 14$

infiltration

Lymphatic metastasis

Nerve/vessel invaded

Not invaded

$\begin{array}{llll}34 & 13 & 0.695 & 0.404\end{array}$

TNM stages

$\begin{array}{lllll}\mathrm{I} / \mathrm{II} & 35 & 10 & 0.109 & 0.751\end{array}$

III/IV

$45 \quad 15$

Distant metastasis

$\begin{array}{lrrrr}\text { Positive } & 6 & 3 & 0.492 & 0.483 \\ \text { Negative } & 74 & 22 & & \end{array}$

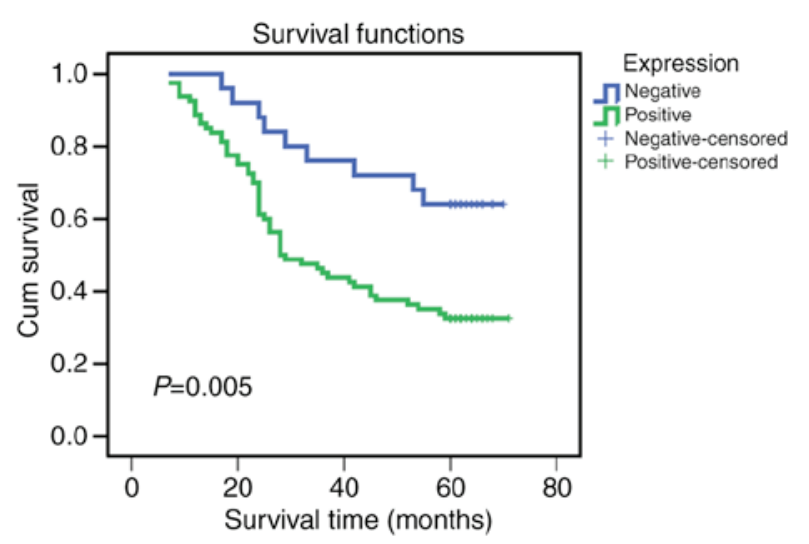

Figure 2. Kaplan-Meier curve of 105 CRC patients with different expression levels of ANXA9 protein. Results of Kaplan-Meier survival analysis indicated that the survival rate of CRC patients with positive ANXA9 protein was lower than that of those with negative ANXA9 protein. CRC, colorectal cancer; ANXA9, Annexin A9.

Effect of ANXA9-siRNA on ANXA9 protein in HCT116 cells. The result of western blotting demonstrated that after a 48 -h 
Table III. Analysis of COX proportional hazards model results in the colorectal cancer patients.

\begin{tabular}{lcccccccc}
\hline Variable & & & & & & & & \multicolumn{2}{c}{ 95\% CI for Exp $(B)$} \\
\cline { 5 - 9 } & $B$ & $S E$ & Wald & $d f$ & Sig & Exp $(B)$ & Lower & Upper \\
ANXA9 expression & 0.992 & 0.432 & 5.273 & 1 & 0.022 & 2.696 & 1.156 & 6.284 \\
Lymphatic metastasis & 0.879 & 0.367 & 5.730 & 1 & 0.017 & 2.409 & 1.173 & 4.950 \\
TNM stages & -1.289 & 0.903 & 2.039 & 1 & 0.153 & 0.276 & 0.047 & 1.617 \\
Invasion & -.066 & 0.862 & 0.006 & 1 & 0.939 & 0.936 & 0.173 & 5.068 \\
Sex & 0.189 & 0.288 & 0.430 & 1 & 0.512 & 1.207 & 0.687 & 2.122 \\
Age (years) & -0.010 & 0.015 & 0.486 & 1 & 0.486 & 0.990 & 0.961 & 1.019 \\
Differentiation & 0.624 & 0.272 & 5.286 & 1 & 0.021 & 1.867 & 1.097 & 3.179 \\
Nerve/vessel & -0.101 & 0.272 & 0.139 & 1 & 0.709 & 0.904 & 0.531 & 1.539 \\
Distant metastasis & 1.000 & 0.450 & 4.949 & 1 & 0.026 & 2.719 & 1.126 & 6.563 \\
\hline
\end{tabular}

B, Coefficient of regression; SE, standard error; Wald, statistic of Wald test coefficient; df, degree of freedom; Sig, P-value; Exp(B), odds ratio; 95\% CI, 95\% confidence interval.

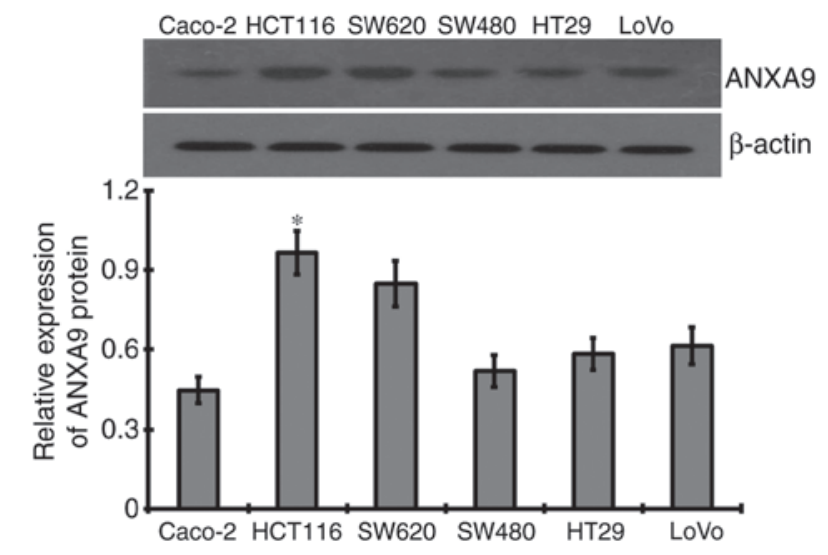

Figure 3. Expression level of ANXA9 protein in CRC cell lines. Different CRC cell lines were selected to evaluate the expression level of ANXA9. The strongest ANXA9 protein was detected in HCT116 cells, and the HCT116 cells were utilized for the in vitro study. ${ }^{*} \mathrm{P}<0.05$ vs. other cell lines. ANXA9, Annexin A9; CRC, colorectal cancer.

transfection with $20 \mu \mathrm{mol} / 1$ ANXA9-siRNA, the ANXA9 expression level was downregulated more significantly when compared with the NS-siRNA and blank groups (Fig. 4).

Impact of ANXA9-siRNA on activity of HCT116 cells. The cell activity of the ANXA9-siRNA group varied with different concentrations and durations (Fig. 5A). Following transfection with ANXA9-siRNA $(20 \mu \mathrm{mol} / 1)$ for $48 \mathrm{~h}$, the cell activity of the ANXA9-siRNA group $(49.64 \pm 5.82 \%)$ was significant lower than that in the NS-siRNA group $(98.62 \pm 9.69 \%)$ and the blank group $(100 \pm 11.24 \% ; \mathrm{P}<0.05)$, as demonstrated in Fig. 5B.

Effect of ANXA9-siRNA on migration and invasion activities in HCT116 cells. Results of the wound healing assay (Fig. 6A) and Transwell assay (Fig. 6B) demonstrate that, following ANXA9-siRNA transfection, the migration and invasion of HCT116 cells treated with ANXA9-siRNA were significantly
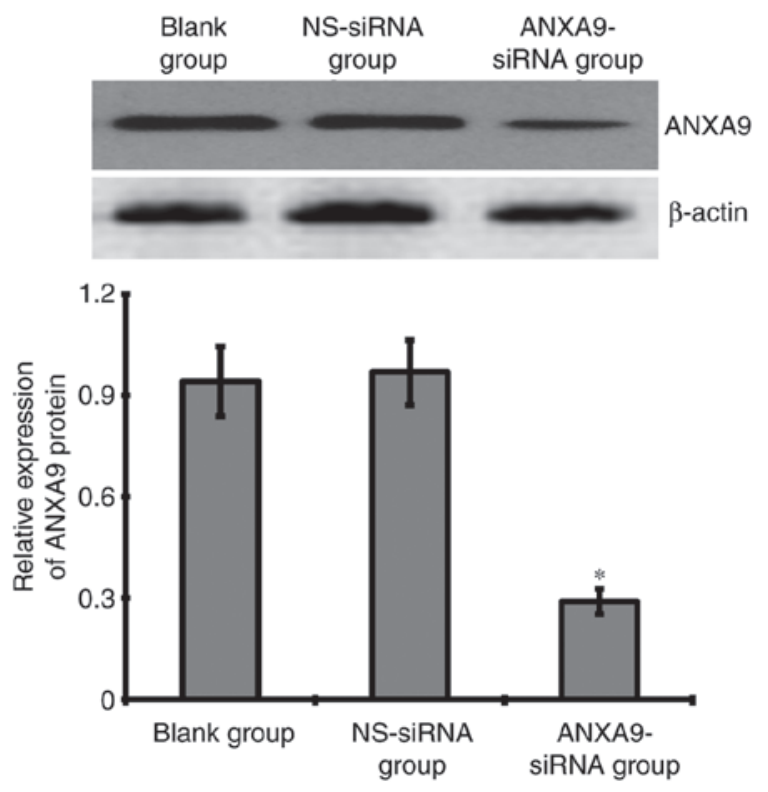

Figure 4. Effect of ANXA9-siRNA on ANXA9 protein expression levels in HCT116 cells. ANXA9-siRNA was transfected into HTC116 cells causing the ANXA9 expression level to be downregulated significantly when compared with the NS-siRNA and blank groups. ${ }^{*} \mathrm{P}<0.05$ vs. blank group or the NS-siRNA group. ANXA9, Annexin A9; CRC, colorectal cancer; non-specific control siRNA.

decreased when compared with the NS-siRNA group and the blank group $(\mathrm{P}<0.05)$, as shown in Fig. 6.

Effect of ANXA9-siRNA on expression levels of ADAM17, MMP-9, TIMP-1, E-cadherin and N-cadherin in HCT116 cells. Expression levels of ADAM17 and MMP-9 mRNA and proteins were significantly downregulated, while TIMP-1 and E-cadherin mRNA and protein expression levels were significantly upregulated in HCT116 cells following ANXA9-siRNA transfection $(\mathrm{P}<0.05)$, and no obvious variation was observed in $\mathrm{N}$-cadherin following ANXA9-siRNA transfection (P>0.05; Fig. 7). 

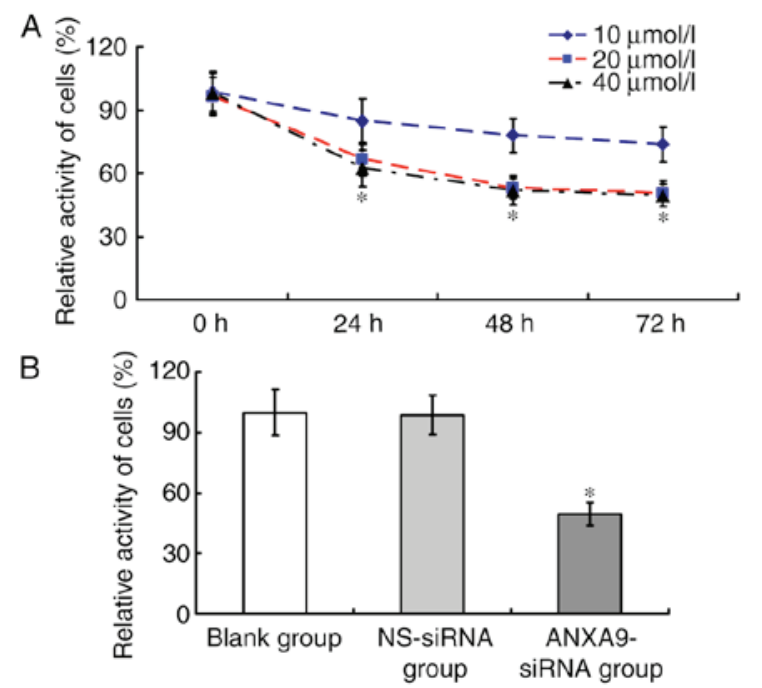

Figure 5. Effect of ANXA9-siRNA on cell activity of HCT116 cells. (A) The activity of HCT116 cells varied with different dosage and duration following ANXA9-siRNA transfection. (B) Compared with the NS-siRNA group and blank group, the activity of HCT116 cells in the ANXA9-siRNA group decreased significantly. "P<0.05 vs. blank group or NS-siRNA group. ANXA9, Annexin A9; CRC, colorectal cancer; non-specific control siRNA.



Figure 6. Effects of ANXA9-siRNA on migration and invasion of HCT116 cells. (A) HCT116 cells were transfected with ANXA9-siRNA and were subjected to wound healing assay to detect cell migrating activity. (B) Transwell assay was utilized to detect cell invasive activity. Results demonstrated that migration and invasion of HCT116 cells decreased following ANXA9-siRNA transfection. ${ }^{*} \mathrm{P}<0.05$ vs. blank group or NS-siRNA group. ANXA9, Annexin A9; non-specific control siRNA.

\section{Discussion}

The incidence of CRC has increased in recent years (14) and has unsatisfactory treatment outcomes. Although certain risk factors of CRC have been identified in terms of diet $(15,16)$, environment (17) and genetics (18), factors that determine the risk of disease remain poorly understood. In the early stages, the symptoms of CRC are often insidious; therefore patients with CRC are typically diagnosed at the advanced stage with a relatively rapid progression and metastases. One of the reasons for the rapid progression of CRC is the strong ability of the cancerous cells to invade and metastasize $(3,4)$. Therefore, suppression of invasion and metastasis of CRC cells may contribute to improvement of the treatment of this illness. Multiple genes and signaling pathways are important in the progression of CRC $(5-8,19,20)$, including various members of the ANXA family. Zhang et al (21) demonstrated that non-steroid anti-inflammatory drugs affect the activity of the nuclear factor- $\mathrm{\kappa B}$ signaling pathway resulting in ANXA1 inhibition, which may lead to growth suppression of CRC cells. In the study by Yang et al (22), ANXA2 was verified to be correlated with the clinicopathological characteristics of CRC. Miyoshi et al (12) reported that a high expression level of ANXA9 mRNA was a marker of poor prognosis for CRC. These studies indicate that ANXAs are significantly associated with CRC development. However, to the best of our knowledge, the association between the ANXA9 gene and CRC has only been examined in one study and only the mRNA expression level of clinical value was reported (12).

In the present study, the clinical value of ANXA9 expression in patients with CRC was investigated in cancer and adjacent mucosa tissue samples (obtained from 105 patients) using IHC. The results demonstrated that a positive ANXA9 expression rate in the cancer tissue samples was higher than that in the mucosal tissue samples. Furthermore, the western blot result was consistent with the IHC result, indicating that ANXA9 may be involved in carcinogenesis development and progression. In addition, further analysis demonstrated that ANXA9 was associated with tumor infiltration depth and lymphatic metastasis, implying that ANXA9 may contribute to CRC invasion and migration. Furthermore, prognostic analysis demonstrated a lower survival rate in the patients with positive ANXA9 protein expression, which was also an independent risk factor for patient survival. These results indicated that ANXA9 protein may be significant in prognostic evaluation, as well as being a marker of poor prognosis with positive expression.

The ANXA9 gene (size, 8,233 bp) is located in human chromosome 1q21, contains 14 exons and encodes 345 amino acid chains $(23,24)$. The association between ANXA9, and CRC cell invasion and migration has not yet been defined. Our further aim is therefore to analyze the function of ANXA9 in CRC invasion and metastasis using RNA interference technology to suppress ANXA9 expression of HCT116 cells in CRC. The present study demonstrated that ANXA9 inhibition resulted in a significant decrease in HCT116 cell proliferation, as well as decreased ability of invasion and migration. In order to understand the molecular mechanism of regulation by ANXA9, the changes of ADAM17, MMP-9 
A

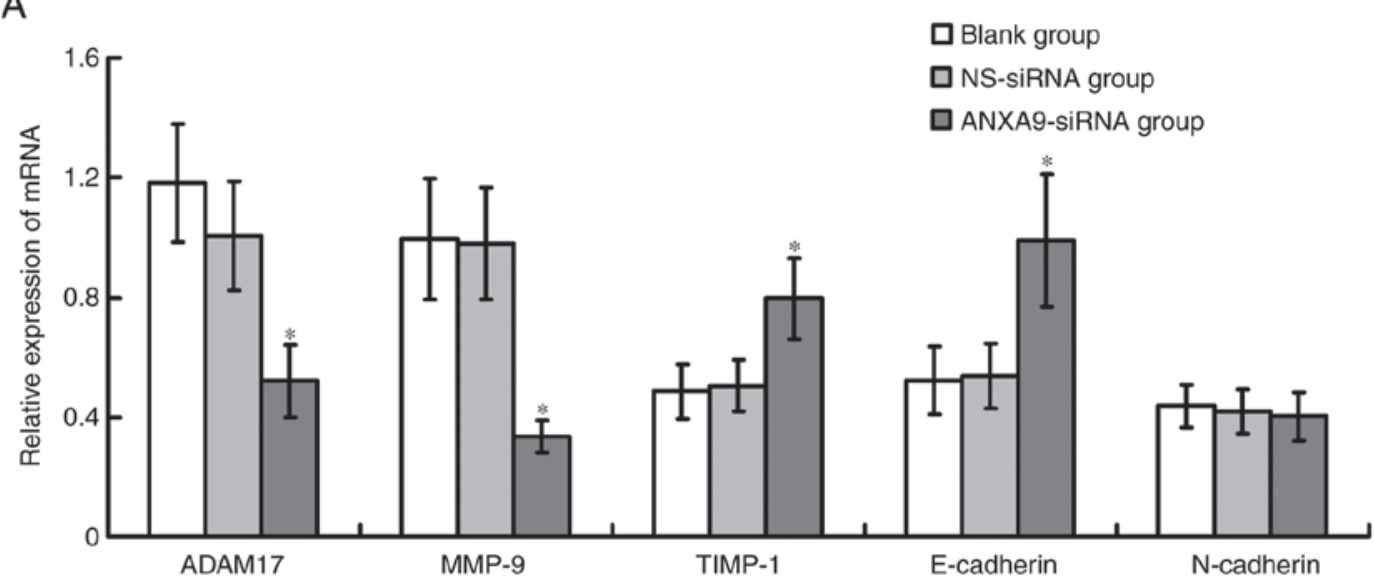

B

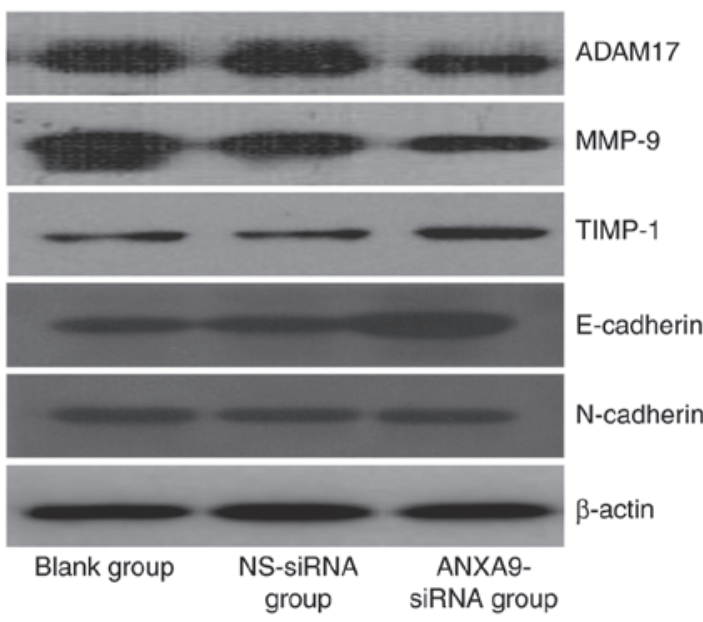

C

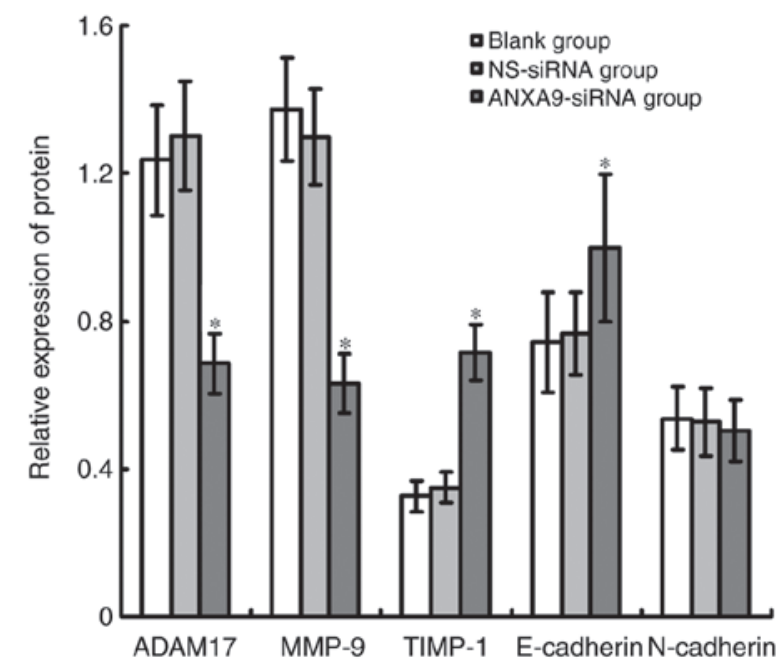

Figure 7. Effect of ANXA9-siRNA on the expression levels of ADAM17, MMP-9 and TIMP-1 genes in HCT116 cells. (A) HCT116 cells were transfected with ANXA9-siRNA, and subjected to quantitative polymerase chain reaction to detect the mRNA expression levels of ADAM17, MMP-9 and TIMP-1. (B and C) HCT116 cells were transfected with ANXA9-siRNA, and subjected to western blot analysis to detect the proteins of ADAM17, MMP-9 and TIMP-1. The expression levels of ADAM17 and MMP-9 decreased, while TIMP-1 expression increased in the HCT116 cells. "P<0.05 vs. blank group or NS-siRNA group. ANXA9, Annexin A9; non-specific control siRNA; ADAM17, ADAM metallopeptidase domain 17; MMP-9, matrix metallopeptidase 9; TIMP-1, tissue inhibitors of metalloproteinases-1.

and TIMP-1 expression levels were detected in HCT116 cells folloing inhibition of ANXA9 expression. ADAM17 is a family members of disintegrin and metalloprotease, of which the upregulated expression in CRC participates in tumor progression (25). Furthermore, a previous study demonstrated that suppression of ADAM17 expression in the CRC cell, MC38CEA results in inhibition of activity and migration (26). MMP-9, an important member of the MMP family, is significant in CRC progression $(27,28)$, whereas TIMP-1 (an MMP-9 suppressor) inhibits MMP-9 and therefore decreases the ability of cancerous cells to invade and migrate $(29,30)$. The present study demonstrated that inhibitation of ANXA9 expression resulted in reduction of ADAM17 expression levels, whereas the level of MMP-9 expression increased in TIMP-1. In addition, ANXA9 was identified to be correlated with ADAM17, where MMP-9 is downregulated by mediation of ADAM17 (31). These results indicate that inhibition of ANXA9 expression levels in HCT116 cells may induce suppression of ADMA17 and
MMP-9 expression levels, but increase the TIMP-1 expression level contributing to the weakness of tumor cells in invasion and migration (26,32). Epithelial-mesenchymal transition (EMT) contributes to the invasion and migration of CRC (33). E-cadherin and N-cadherin are important in EMT of CRC $(34,35)$. The results of the current study demonstrate that E-cadherin was upregulated after ANXA9 inhibition, whereas no obvious variation was verified in N-cadherin. To better understand the association between ANXA9 and CRC, further studies at the molecular level are required.

In conclusion, the present study demonstrates that ANXA9 may be a novel marker of poor prognosis. Inhibition of ANXA9 expression may suppress the activity, invasion and metastasis of CRC cells by regulating ADAM17, MMP-9, TIMP-1 and E-cadherin. This indicates that ANXA9 may be associated with invasion and metastasis of CRC. Thus, the current study may provide evidence for further research into CRC development, prognostic markers and gene targeted therapeutic strategies. 


\section{Competing interests}

The authors declare that they have no competing interests.

\section{References}

1. Schreuders EH, Ruco A, Rabeneck L, Schoen RE, Sung JJ, Young GP and Kuipers EJ: Colorectal cancer screening: A global overview of existing programmes. Gut 64:1637-1649, 2015.

2. Mol L, Ottevanger PB, Koopman M and Punt CJ: The prognostic value of WHO performance status in relation to quality of life in advanced colorectal cancer patients. Eur J Cancer 66: 138-143, 2016.

3. van Wyk HC, Roxburgh CS, Horgan PG, Foulis AF and McMillan DC: The detection and role of lymphatic and blood vessel invasion in predicting survival in pa'tients with node negative operable primary colorectal cancer. Crit Rev Oncol Hematol 90: 77-90, 2014.

4. Ning Y and Lenz HJ: Targeting IL-8 in colorectal cancer. Expert Opin Ther Targets 16: 491-497, 2012.

5. Wu M, Wang J, Tang W, Zhan X, Li Y, Peng Y, Huang X, Bai Y, Zhao J, Li A, et al: FOXK1 interaction with FHL2 promotes proliferation, invasion and metastasis in colorectal cancer Oncogenesis 5: e271, 2016.

6. Jeong SH, Jeon YJ and Park SJ: Inhibitory effects of dieckol on hypoxia-induced epithelial-mesenchymal transition of HT29 human colorectal cancer cells. Mol Med Rep 14: 5148-5154, 2016.

7. Kim BR, Kang MH, Kim JL, Na YJ, Park SH, Lee SI, Kang S, Joung SY, Lee SY, Lee DH, et al: RUNX3 inhibits the metastasis and angiogenesis of colorectal cancer. Oncol Rep 36: 2601-2608, 2016.

8. Shi W, Ye Z, Zhuang L, Li Y, Shuai W, Zuo Z, Mao X, Liu R, $\mathrm{Wu}$ J, Chen $\mathrm{S}$ and Huang W: Olfactomedin 1 negatively regulates $\mathrm{NF}-\mathrm{KB}$ signalling and suppresses the growth and metastasis of colorectal cancer cells. J Pathol 240: 352-365, 2016.

9. Boudhraa Z, Bouchon B, Viallard C, D'Incan M and Degoul F: Annexin A1 localization and its relevance to cancer. Clin Sci 130: 205-220, 2016

10. Qi H, Liu S, Guo C, Wang J, Greenaway FT and Sun MZ: Role of Annexin A6 in cancer. Oncol Lett 10: 1947-1952, 2015.

11. Lauritzen SP, Boye TL and Nylandsted J: Annexins are instrumental for efficient plasma membrane repair in cancer cells. Semin Cell Dev Biol 45: 32-38, 2015.

12. Miyoshi N, Yamamoto H, Mimori K, Yamashita S, Miyazaki S, Nakagawa S, Ishii H, Noura S, Ohue M, Yano M, et al: ANXA9 gene expression in colorectal cancer: A novel marker for prognosis. Oncol Lett 8: 2313-2317, 2014.

13. Edge SB and Compton CC: The American Joint Committee on Cancer: The 7th edition of the AJCC cancer staging manual and the future of TNM. Ann Surg Oncol 17: 1471-1474, 2010

14. Bode AM, Dong Z and Wang H: Cancer prevention and control: Alarming challenges in China. Natl Sci Rev 3: 117-127, 2016.

15. Liu J, Zhou Q, Xu J, Wang J and Zhang Y: Detection of EGFR expression in patients with colorectal cancer and the therapeutic effect of cetuximab. J BUON 21: 95-100, 2016.

16. Bhopal RS: Diet and colorectal cancer incidence. JAMA Intern Med 175: 1726-1727, 2015.

17. Marley AR and Nan H: Epidemiology of colorectal cancer. Int J Mol Epidemiol Genet 7: 105-114, 2016.

18. Cai Z, Han S, Li Z, He L, Zhou J, Huang W and Xu Y: A genome-wide assessment of variations of primary colorectal cancer maintained in metastases. Gene 595: 18-24, 2016.
19. Wang H, Xi Q and Wu G: Fatty acid synthase regulates invasion and metastasis of colorectal cancer via Wnt signaling pathway. Cancer Med 5: 1599-1606, 2016.

20. Zou F, Mao R, Yang L, Lin S, Lei K, Zheng Y, Ding Y, Zhang P, Cai G, Liang X and Liu J: Targeted deletion of miR-139-5p activates MAPK, NF- $\kappa \mathrm{B}$ and STAT3 signaling and promotes intestinal inflammation and colorectal cancer. FEBS J 283: 1438-1452, 2016.

21. Zhang Z, Huang L, Zhao W and Rigas B: Annexin 1 induced by anti-inflammatory drugs binds to NF-kappaB and inhibits its activation: Anticancer effects in vitro and in vivo. Cancer Res 70: 2379-2388, 2010.

22. Yang T, Peng H, Wang J, Yang J, Nice EC, Xie K and Huang C: Prognostic and diagnostic significance of Annexin A2 in colorectal cancer. Colorectal Dis 15: e373-e381, 2013.

23. Boczonadi V and Määttä A: Annexin A9 is a periplakin interacting partner in membrane-targeted cytoskeletal linker protein complexes. FEBS Lett 586: 3090-3096, 2012

24. Goebeler V, Ruhe D, Gerke V and Rescher U: Atypical properties displayed by annexin A9, a novel member of the annexin family of $\mathrm{Ca}^{2+}$ and lipid binding proteins. FEBS Lett 546: 359-364, 2003.

25. Blanchot-Jossic F, Jarry A, Masson D, Bach-Ngohou K, Paineau J, Denis MG, Laboisse CL and Mosnier JF: Up-regulated expression of ADAM17 in human colon carcinoma: Co-expression with EGFR in neoplastic and endothelial cells. J Pathol 207: 156-163, 2005.

26. Das S, Czarnek M, Bzowska M, Mężyk-Kopeć R, Stalińska K, Wyroba B, Sroka J, Jucha J, Deneka D, Stokłosa P, et al: ADAM17 silencing in mouse colon carcinoma cells: The effect on tumoricidal cytokines and angiogenesis. PLoS One 7: e50791, 2012.

27. Lima AI, Mota J, Monteiro SA and Ferreira RM: Legume seeds and colorectal cancer revisited: Protease inhibitors reduce MMP-9 activity and colon cancer cell migration. Food Chem 197: 30-38, 2016

28. Liu F, Zhang T, Zou S, Jiang B and Hua D: B7-H3 promotes cell migration and invasion through the Jak2/Stat3/MMP9 signaling pathway in colorectal cancer. Mol Med Rep 12: 5455-5460, 2015.

29. Weidle UH, Birzele F and Krüger A: Molecular targets and pathways involved in liver metastasis of colorectal cancer. Clin Exp Metastasis 32: 623-635, 2015

30. Christensen IJ, Brünner N, Dowell B, Davis G, Nielsen HJ, Newstead G and King D: Plasma TIMP-1 and CEA as markers for detection of primary colorectal cancer: A prospective validation study including symptomatic and non-symptomatic individuals. Anticancer Res 35: 4935-4941, 2015.

31. Nakayama H,Fukuda S, Inoue H, Nishida-Fukuda H, Shirakata Y, Hashimoto K and Higashiyama S: Cell surface annexins regulate ADAM-mediated ectodomain shedding of proamphiregulin. Mol Biol Cell 23: 1964-1975, 2012.

32. Xiao LJ, Lin P, Lin F, Liu X, Qin W, Zou HF, Guo L, Liu W, Wang SJ and Yu XG: ADAM17 targets MMP-2 and MMP-9 via EGFR-MEK-ERK pathway activation to promote prostate cancer cell invasion. Int J Oncol 40: 1714-1724, 2012.

33. Li Q, Wang Y, Lai Y, Xu P and Yang Z: HspB5 correlates with poor prognosis in colorectal cancer and prompts epithelial-mesenchymal transition through ERK signaling. PLoS One 12: e0182588, 2017.

34. Iseki Y, Shibutani M, Maeda K, Nagahara H, Ikeya T and Hirakawa K: Significance of E-cadherin and CD44 expression in patients with unresectble metastatic colorectal cancer. Oncol Lett 14: 1025-1034, 2017.

35. Yan X, Yan L, Liu S, Shan Z, Tian Y and Jin Z: N-cadherin, a novel prognostic biomarker, drives malignant progression of colorectal cancer. Mol Med Rep 12: 2999-3006, 2015. 